\title{
Complement depletion during haemofiltration with polyacrilonitrile membranes
}

\author{
Yvan Gasche, Manuel Pascual ${ }^{1}$, Peter M. Suter, Hervé Favre ${ }^{1}$, Jean-Claude Chevrolet $^{2}$ and \\ Jürg A. Schifferli ${ }^{1}$
}

Division of Surgical Intensive Care, Department of Anaesthesiology, ${ }^{1}$ Division of Nephrology, Department of Medicine and ${ }^{2}$ Division of Medical Intensive Care, Department of Medicine, Hôpital Cantonal, Universitaire de Genève, Geneva, Switzerland

\begin{abstract}
Background. Polyacrylonitrile (PAN, AN69 ${ }^{(1)}$ ) dialysis membranes have been shown to improve the outcome of critically ill patients. Factor D is an essential enzyme of the alternative pathway of complement and is increased during renal failure. On the other hand the contact of blood with biomaterials activates the complement cascade through the alternative pathway. PAN filters adsorb factor D which looses its enzymatic activity whilst bound to the membrane [1]; the complement alternative pathway function of serum exposed to PAN filters is greatly diminished and restored after addition of purified factor D [1]. The aim of our study was to measure the time course of factor $\mathrm{D}$ adsorption and its blood concentration during CVVH in critically ill patients with acute renal failure.

Methods. We studied seven critically ill patients with ARF before, during and after continuous veno-venous haemofiltration (CVVH) with AN69.

Results. There was a rapid decrease of factor $D$ levels to $62( \pm 6 \%)$ of the pre-CVVH value during the first $2 \mathrm{~h}$, which continued to $51( \pm 7.3 \%)$ after $12 \mathrm{~h}$; at $24 \mathrm{~h}$ there was a slight rise to $62 \pm 12 \%$. Sequential use of Polyacrylonitrile (AN69 ${ }^{\text {(日) }}$ ) filters lowered factor D levels below the normal plasma concentration in three patients, thus producing a state of factor D depletion. Conclusion. The significant reduction of factor $D$ levels during CVVH with PAN filters suggests that frequent changes of PAN filters may reduce alternative pathway function by lowering factor D levels. CVVH (as opposed to intermittent dialysis) with PAN membranes may further improve the outcome of critically ill patients.
\end{abstract}

Key words: acute renal failure; CVVH; factor D; PAN (AN69 ${ }^{\text {B }}$ ) filters

Correspondence and offprint requests to: Peter M. Suter, Professor and Chief, Division of Surgical Intensive Care, Department of Anaesthesiology, Hopital Cantonal, Universitaire de Genéve, Geneva, Switzerland.

\section{Introduction}

Recently, a prospective randomized study indicated that the outcome of critically ill patients was improved by biocompatible membranes made of polyacrylonitrile (AN69 ${ }^{\circ}$ ) during discontinuous haemodialysis, as compared with cuprophane membranes [2]. The adsorptive properties of PAN membrane are crucial to determine its biocompatibility with regard to complement activation. In particular, the anaphylatoxins C $3 a$ and C5a, as well as complement factor D are adsorbed very efficiently by $A N 69^{\infty}[1]$. Factor $D$ is the rate limiting enzyme of the alternative pathway of complement (AP) activation. Its plasma concentration is low in normal individuals $(2 \mu \mathrm{g} / \mathrm{ml})$, but is increased approximately ten-fold in patients with end-stage renal failure, due to a decreased renal catabolism $[3,4]$. The increased level of factor $D$ in patients with renal failure is directly responsible for an enhanced activity of the AP, which may be detrimental [5]. Factor $\mathrm{D}$ removal by adsorption on PAN (AN69 ${ }^{\circledR}$ ) has been shown to be substantial, with $80 \%$ reduction of plasma levels after regular haemodialysis sessions [1]. Thus PAN membranes have the potential to deplete factor $\mathrm{D}$ from the circulation and possibly down regulate complement activation. We studied the efficiency of factor D removal by AN69 filters during continuous veno-venous haemofiltration in critically ill patients with acute renal failure.

\section{Methods}

Seven critically ill patients with ARF requiring CVVH were studied (mean age: 62.1 years; range: $41-81$ ). The aetiologies of ARF were septic shock $(n=3)$, haemodynamic insufficiency due to cardiac failure $(n=3)$, and hepatorenal syndrome $(n=1)$. The hollow-fibre dialysis filters were replaced after clotting or because of insufficient ultrafiltration rate $(<700 \mathrm{ml} / \mathrm{h})$. CVVH was performed with blood flows fixed at $250 \mathrm{ml} / \mathrm{min}$. AN6 ${ }^{\$}$ filters (copolymers of polyacrylonitrile and sodium metallylsulfonate, Hospal, Lyon, France) had an effective surface of 0.6 or $0.9 \mathrm{~m}^{2}$ (Multiflow 60 or 100 , respectively), the mean ultrafiltration rates were $1000 \propto / \mathrm{h}$ 
for the eight $0.6 \mathrm{~m}^{2}$ and $1500 \mathrm{c} / \mathrm{h}$ for the six $0.9 \mathrm{~m}^{2}$ filters studied; in addition, filters made of polyamide fibres (Gambro, FH66, Hechingen, Germany) were also studied and used as controls. Their effective surface of ultrafiltration was $0.6 \mathrm{~m}^{2}$ and the mean ultrafiltration rate was $1500 \propto / \mathrm{h}$ for the five filters studied.

Blood samples were collected on EDTA before starting CVVH and during the procedure at 15 min., $1,2,4,6,12$ and $24 \mathrm{~h}$ in the arterial and venous lines. Plasma was immediately separated by centrifugation at $1500 \mathrm{~g}$ for $10 \mathrm{~min}$ at $4^{\circ} \mathrm{C}$. Ultrafiltrate was collected simultaneously without additive. All samples were stored at $-80^{\circ} \mathrm{C}$. Factor D haemolytic activity was determined as previously described [3]. IgM levels were measured by laser nephelometry and used to correct results for possible changes in haemoconcentration during the CVVH procedure.

No patient received whole blood or plasma during the studies.

The results are given as mean ( \pm standard error of the mean) and statistical analysis are performed using analysis of variance (ANOVA).

\section{Results}

The levels of factor D before CVVH differed from one patient to another according to the severity and

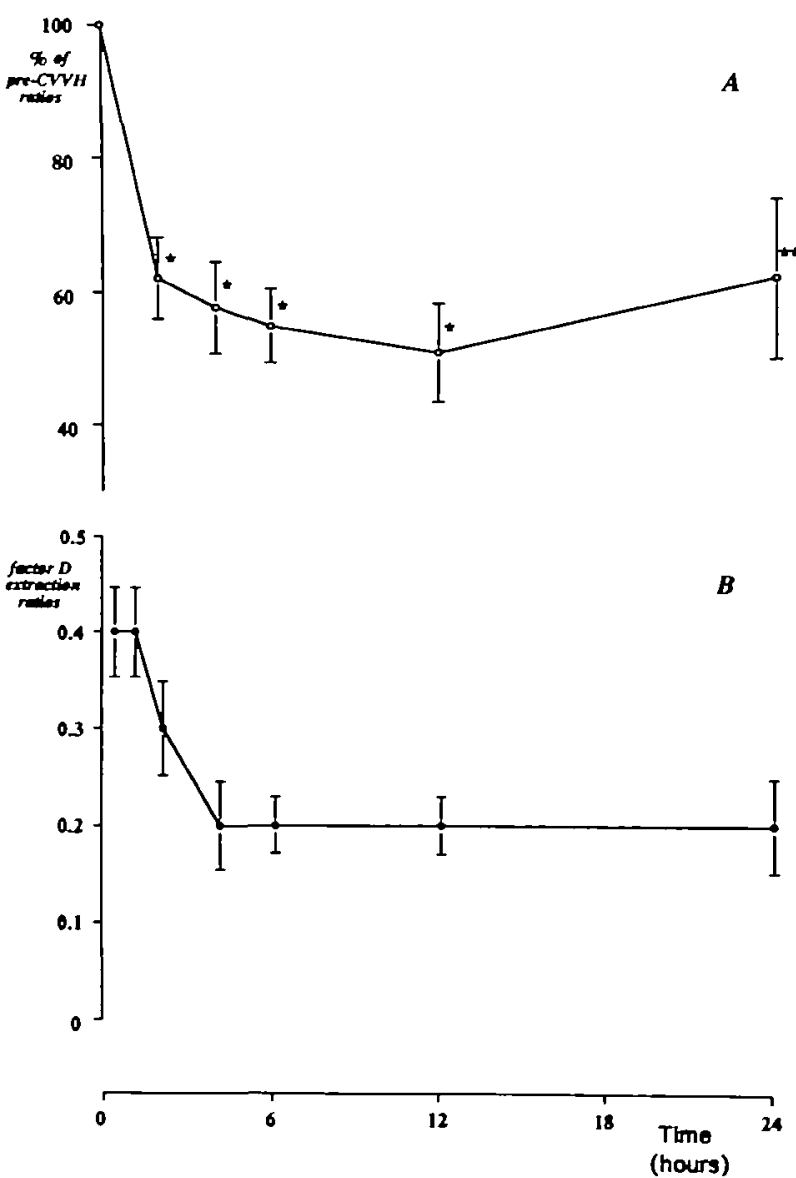

Fig. 1. A. Mean ( \pm SEM) factor $D$ change in percent of pre-CVVH ratios with 14 AN69 filters at $2 \mathrm{~h}, 4 \mathrm{~h}, 6 \mathrm{~h}, 12 \mathrm{~h}$, and $24 \mathrm{~h}$ of CVVH. ANOVA for repeated measures between pre-CVVH ratio and $2 \mathrm{~h}$ $4 \mathrm{~h}, 6 \mathrm{~h}, 12 \mathrm{~h}, 24 \mathrm{~h}$ ratios: ${ }^{*} P<0.0001$; ${ }^{* *} P<0.0026$. B. Mean ( \pm SEM) factor $D$ extraction ratios (pre-filter-post-filter/pre-filter samples) at $15 \mathrm{~min}, 1 \mathrm{~h}, 2 \mathrm{~h}, 4 \mathrm{~h}, 6 \mathrm{~h}, 12 \mathrm{~h}$ and $24 \mathrm{~h}$ of CVVH. duration of renal failure (mean: $7 \mu \mathrm{g} / \mathrm{ml}$; ranges: $2.3-11.1 \mu \mathrm{g} / \mathrm{ml}$ ). CVVH with PAN membranes was followed by an immediate fall in the levels of factor $D$ in all patients. The drop was fast during the first two hours and slower over the following $10 \mathrm{~h}$. In patients in whom the dialysis filters were not changed $(n=2)$, factor $D$ increased slightly over the next $12 \mathrm{~h}$ (Figure 1A). The extraction of factor D by AN69 filters was calculated from the pre- and post-filter difference. As expected from the changes in factor $D$ levels, the extraction was highest during the first hours, then levelled off during the period of time studied (Figure 1B). There was still a measurable extraction at $24 \mathrm{~h}$, at a time of rising factor D levels in plasma. Interestingly it was possible in three patients to lower factor $D$ concentrations below the mean values of normal individuals $(2 \mu \mathrm{g} / \mathrm{ml})$ by using two PAN filters sequentially, as illustrated for one patient in Figure 2. Factor D increased immediately upon interruption of haemofiltration even for the short period of time required to change from one PAN filter to the next (Figures 2 and 3). The immediate effects of $\mathrm{CVVH}$ with PAN were also evident when the control polyamide filters were used either before or after the PAN membranes (Figures 2 and 3). No factor D was found in the ultrafiltrate of PAN filters during the first $12 \mathrm{~h}$

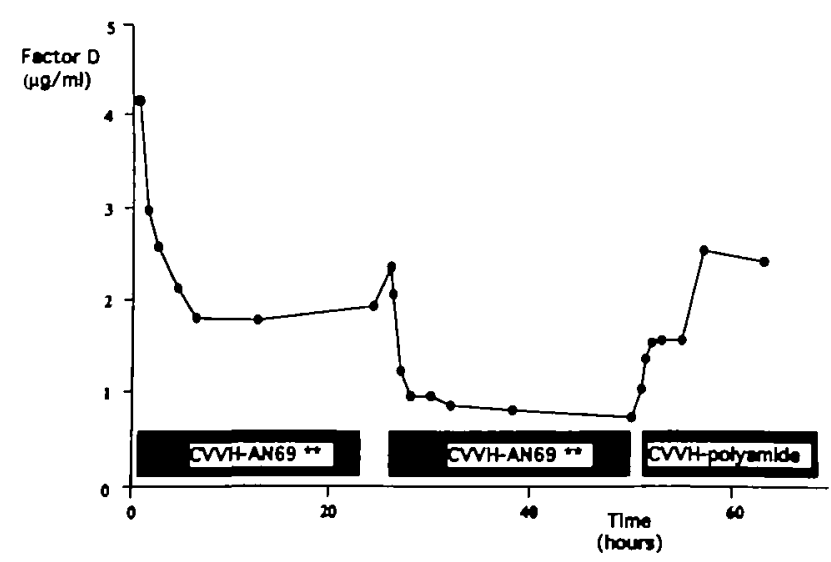

Fig. 2. Plasma levels of factor $\mathrm{D}(\mu \mathrm{g} / \mathrm{ml})$ during CVVH with small AN69 (AN69**) and polyamide filters in patient 1.

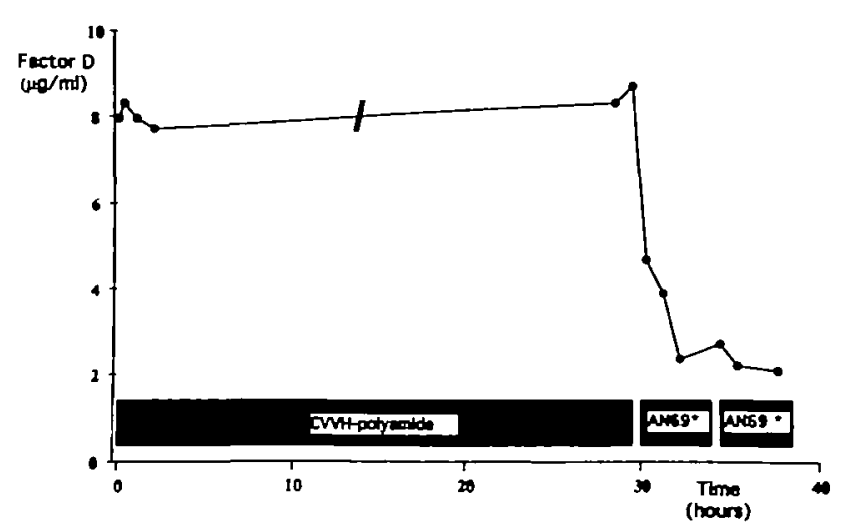

Fig. 3. Plasma levels of factor $\mathrm{D}(\mu \mathrm{g} / \mathrm{ml})$ during CVVH with polyamide and large AN69 (AN69*) filters in patient 2. 
and only minimal amounts $(10-20 \mu \mathrm{g} / 1)$ could be detected at $24 \mathrm{~h}$. Four PAN membranes $(2$ small and 2 large) were washed immediately after use with $15 \mathrm{mM}$ $\mathrm{NaCl}$, followed by elution of membrane associated proteins with $2 \mathrm{M} \mathrm{NaCl}[1]$.

Quantitative analysis indicated that 52 and $53 \mathrm{mg}$ of factor $\mathrm{D}$ were eluted from two $0.6 \mathrm{~m}^{2}$ filters, and 66 and $60 \mathrm{mg}$ from two $0.9 \mathrm{~m}^{2}$ filters, respectively. No factor $\mathrm{D}$ could be eluted from polyamide membranes using similar conditions.

\section{Discussion}

The main finding in this study was that CVVH with PAN (AN69 ) filters lowered factor D levels very significantly, producing in some patients a state of factor D depletion. Removal of factor $D$ was mainly due to adsorption to the PAN membrane, a mechanism which remained efficient for several hours.

Complement activation has been shown to participate in the pathogenesis of sepsis and ARDS and to retard the resolution of ischemic renal failure. Conversely, complement blockade or depletion was beneficial in different animal models of sepsis-ARDS, and dialysis with PAN (as opposed to cuprophane) was associated with faster recovery from ARF in the rate $[6,7,8]$. In vivo, a specific blockade of factor $D$ activity inhibits complement activation by the alternative pathway [9]. PAN membranes both inhibit complement activation at the blood-membrane interface and deplete factor $D$ from plasma [1]. Thus, the adsorption of factor D by PAN membranes may be beneficial in critically ill patients requiring dialysis [2], inspite of a possible increased risk of infection due to alternative pathway inhibition.

In addition, lowering complement activation with PAN membranes may decrease the production of cytokines in these patients, since complement products have been shown to trigger TNF and ILl release by mononuclear cells [10].

In clinical situations such as sepsis with ARF, the PAN membrane should be viewed as a drug, suitable for a definite time, especially if a series of noxious mediators in plasma were down regulated, e.g. complement and cytokines.

Whether CVVH with PAN is superior to intermittent dialysis in the outcome of critically ill patients remains to be studied. If so, frequent changes of PAN filters, or new devices with similar biomaterials may reveal critical for the optimal management of such patients.

\section{References}

1. Pascual M, Schifferli JA. Adsorption of complement factor D by polyacrylonitrile dialysis membranes. Kidney Int 1993; 43: 903-911

2. Schiff H, Lang SM, Konig A, Strasser T, Halder MC, Held E. Biocompatible membranes in acute renal failure: prospective case-controlled study. Lancet 1994; 344: 570-572

3. Pascual M, Steiger G, Estreicher J, Macon K, Volanakis JE, Schifferli JA. Metabolism of complement factor D in renal failure. Kidney Int 1988; 34: 529-536

4. Volanakis JE, Barnum SR, Giddens M, Galla JH. Renal filtration and catabolısm of complement protein D. $N$ Engl $J$ Med 1985; 312: 395-399

5. Pascual M, Paccaud JP, Macon K, Volanakis JE, Schifferli JA. Complement activation by the alternative pathway is modified in renal failure: the role of factor D. Clin Nephrol 1989; 32: $185-193$

6. Stevens JH, O'Hanley P, Shapiro JM et al. Effects of anti-C5a antibodies on the ARDS in septic primates. $J$ Clin Invest 1986; 77: $1812-1816$

7. Rabinovici R, Yeh CG, Hillegass LM, Griswold DE et al. Role of complement in endotoxin/platelet-activating factor induced lung injury. $J$ Immunol 1992; 149: 1744-1750

8. Schulman G, Fogo A, Gung A, Badr K, Hakim R. Complement activation retards resolution of acute ischemic renal failure in the rat. Kidney Int 1991; 40: 1069-1074

9. Pascual M, Catana M, White T, Spiegelman BM, Schifferli JA. Inhibition of complement alternative pathway in mice with Fab antibody to recombinant adipsin/factor D. Eur J Immunol 1993; 23: $1389-1392$

10. Pereira BJG, Dinarello CA. Production of cytokines and cytokine inhibitory proteins in patients on dialysis. Nephrol Dial Transplant 1994; 9 [Suppl. 2]: 60-71 